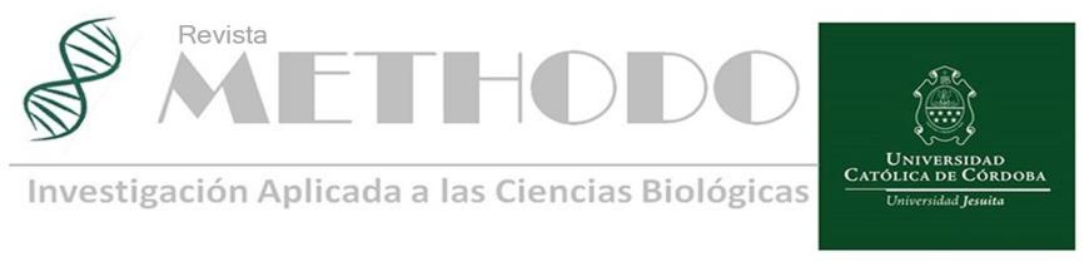

CASO CLINICO Rev. Methodo 2020;5(4):165-168 https://doi.org/10.22529/me.2020.5(4)08

Recibido 31 Oct. 2019 | Aceptado 30 Jul 2020 |Publicado 05 Oct. 2020

\title{
Válvula Aórtica Cuadricúspide: Presentación de un caso Clínico y revisión de literatura
}

\section{Quadricuspid Aortic Valve: Presentation of a clinical case and literature review}

\author{
Sofía P Sanchez ${ }^{1}$ Juan P Gassinoํㅜ, Raúl J Barcudi ${ }^{1}$ \\ 1. Universidad Católica de Córdoba. Facultad de Ciencias de la Salud. Clínica Universitaria Reina Fabiola, Servicio de Cardiología \\ 2. Universidad Católica de Córdoba. Facultad de Ciencias de la Salud. Clínica Universitaria Reina Fabiola, Servicio de Cardiología. \\ 3. Universidad Católica de Córdoba. Facultad de Ciencias de la Salud. Clínica Universitaria Reina Fabiola, Servicio de Cardiología. \\ Correspondencia: Sofia Paola Sánchez. Servicio de Cardiología Clínica Universitaria Reina Fabiola Oncativo 1248-X5004FHP-Córdoba, Argentina. Email: \\ sofia.p.sanchezf@gmail.com
}

\section{Resumen}

Mujer de 51 años que concurre a consultorio para control médico anual, en examen físico se constata soplo regurgitante en foco aórtico por lo que se solicita ecocardiograma transtorácico donde se visualiza una válvula aórtica cuadricúspide.

Palabras clave: válvulas semilunares anormales, válvula aórtica cuadricúspide, insuficiencia aortica asintomática.

\begin{abstract}
A 51-year-old woman comes to the office for annual medical control. A physical examination reveals a regurgitant murmur in the aortic focus. A transthoracic echocardiogram is requested, where a quadricuspid aortic valve is visualized.
\end{abstract}

Keywords: abnormal semilunar valves, quadricuspid aortic valve, asymptomatic aortic regurgitation.

\section{Introducción}

La válvula aórtica cuadricúspide es una variante rara de desarrollo anormal de válvulas semilunares. La incidencia reportada es $0.008 \%$ en series de autopsias, $0.043 \%$ por ecocardiograma transtorácico y $1 \%$ de los publicados con requerimiento de cirugía valvular ${ }^{1-2}$.

El hallazgo más frecuente es la insuficiencia aórtica, con una incidencia de alrededor del $75 \%$ de los casos, y la ocurrencia de estenosis es extremadamente rara $(0,7 \%)^{3}$.

Una clara clasificación de la anatomía de la aorta cuadricúspide es difícil, pero de gran importancia clínica ya que estos pacientes pueden presentar insuficiencia valvular aórtica progresiva y otras anormalidades cardiovasculare ${ }^{4-5}$. Se reportará un caso de válvula aórtica cuadricúspide en un paciente asintomático con soplo cardíaco regurgitante en control de rutina.

\section{Caso clínico}

Se presenta por consultorio externo paciente de sexo femenino de 51 años asintomática para control médico anual, niega antecedentes 
personales patológicos, al interrogatorio refiere soplo de la infancia que persiste en la edad adulta. Al examen físico a la auscultación se constatan ruidos cardiacos normofoneticos con soplo regurgitante en foco aórtico no irradiado $2 / 6$, signos vitales normales con una PA $120 / 80$ y FC $70 \mathrm{lpm}$. Resto del examen sin particularidades.

Electrocardiograma con ritmo sinusal, dentro de parámetros de normalidad.

Se solicita Ecocardiograma donde revela en registro bidimensional válvula aórtica cuadricúspide morfología tipo D (Figura 1), insuficiencia moderada valorada por un tiempo de hemipresión de $382 \mathrm{~ms}$ (Figura 2), ancho del jet por Doppler color en tracto de salida de ventrículo izquierdo de 50\%, volumen aproximado regurgitante de $40 \mathrm{ml}$, Vmax. Ao: $1.9 \mathrm{~m} / \mathrm{seg}$.

No observándose flujo holodiastólico reverso en aorta abdominal.

Función ventricular normal con FE 60\%. Masa ventricular izquierda de $140 \mathrm{gr} / \mathrm{m} 2$ con un espesor parietal de 0.36 .

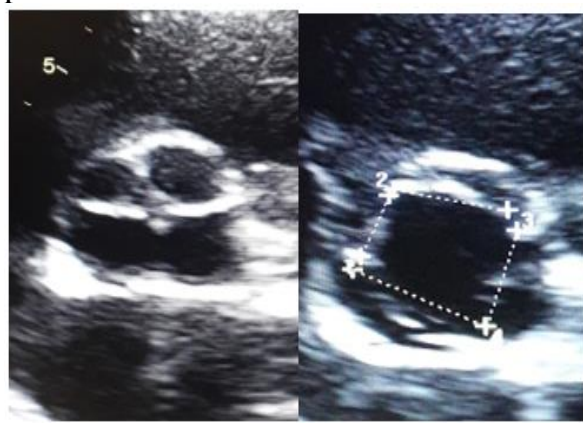

$1 \mathrm{~L} 1.2 \mathrm{~cm}$ $2 \mathrm{~L} 1.7 \mathrm{~cm}$ $3 L 1.7 \mathrm{~cm}$ $4 \mathrm{~L} 2.1 \mathrm{~cm}$

Figura 1. Vista ecocardiográfica de eje paraesterna corto mostrando morfología y dimensiones de la válvula cuadricúspide.

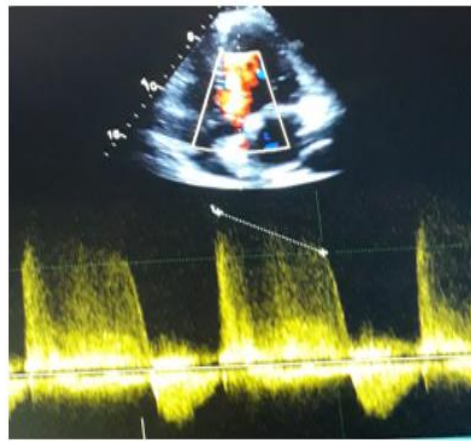

Vmax: 4.75 GPmax: 90.28 TMP 382 Pendiente 3.6

Figura 2. Vista ecocardiográfica de 5 cámaras donde se visualiza insuficiencia aórtica moderada registrada por un tiempo de hemipresión de $382 \mathrm{~ms}$.

\section{Consideraciones embriológicas}

En la quinta semana de embriogénesis normal, se forman dos crestas mesenquimales en la porción cefálica del tronco arterioso. Estas crestas troncales se fusionan y descienden en espiral hacia los ventrículos para formar el tabique aorticopulmonar. En la unión del cono y el tronco, tres hinchazones mesenquimales dan lugar a cada válvula semilunar. Estas inflamaciones crecen para formar válvulas de forma triangular que, con su endotelio de cobertura, se excavan en su aspecto distal para formar las cúspides. El mecanismo que conduce al desarrollo de esta malformación congénita no se conoce bien. La fusión aberrante del tabique aortopulmonar o la proliferación mesenquimatosa anormal en el tronco común puede conducir a la formación anormal de cúspides. La válvula aórtica cuadricúspide puede representar una variante supranumeraria de un brote mesenquimatoso aórtico primario o una proliferación anormal de las cúspides ${ }^{6}$.

\section{Clasificación}

Sobre la base de la variación en el tamaño de las cúspides aórticas, Hurwitz y Roberts7 han clasificado las válvulas aórticas cuadricúspides en siete tipos (Figura 3)

a. Las cuatro cúspides de igual tamaño;

b. Tres cúspides de igual tamaño, la cuarta cúspide de menor tamaño (tipo más común);

c. Dos cúspides más grandes y dos cúspides más pequeñas;

d. Una cúspide más grande, dos cúspides de tamaño intermedio y una cúspide más pequeña

e. Tres cúspides de igual tamaño y una de mayor tamaño;

f. Dos cúspides de igual tamaño y dos cúspides de desigual tamaño más pequeño;

g. Los cuatro tamaños desiguales (la variante menos frecuente).

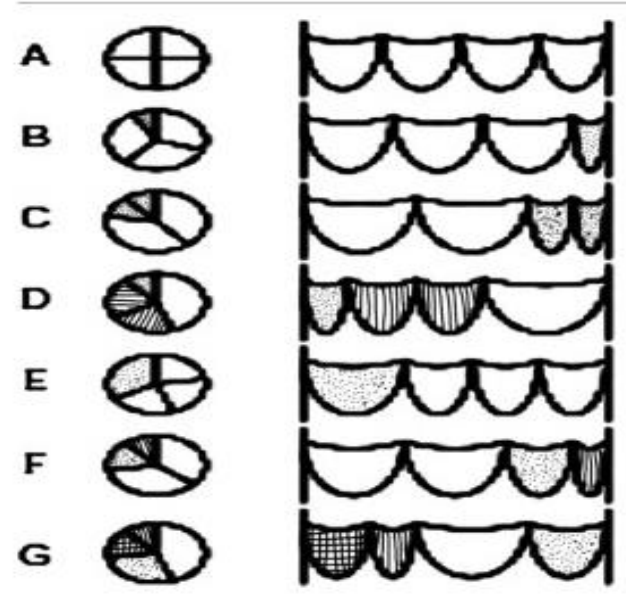

Figura 3. Clasificación de Hurwitz y Roberts de la válvula aórtica cuadricúspide con Vali et al.

Hasta la fecha, se han publicado tres series principales y muchos casos aislados para un total de 271 pacientes; Todos los casos se clasifican de la misma manera. Los tipos A, B y C están

Revista Methodo: Investigación Aplicada a las Ciencias Biológicas. Universidad Católica de Córdoba. Jacinto Ríos 571 Bo Gral. Paz. X5004FXS. Córdoba. Argentina. Tel.: (54) 3514517299 / Correo: methodo@ucc.edu.ar / Web: methodo.ucc.edu.ar | CASO CLINICO Rev. Methodo 2020;5(4):165-168. 
representados en más del $85 \%$; mientras que la variante tipo D es muy rara $^{8}$.

\section{Anomalías asociadas}

La válvula aórtica cuadricúspide se asocia con otras anomalías congénitas en sintonía del 18,3\%. Estas incluyen anomalías de las arterias coronarias, comunicación interventricular, conducto arterioso persistente, estenosis pulmonar, rotura del seno de valsalva, bloqueo cardíaco completo y miocardiopatía hipertrófica ${ }^{3}$. La válvula aórtica cuadricúspide también se ha reportado en asociación con el síndrome de EhlersDanlos ${ }^{9}$. La asociación con arterias coronarias anómalas $(10 \% \text { de los casos })^{3}$, en particular arteria coronaria única, es especialmente importante ya que se ha informado muerte cardíaca repentina causada por el aislamiento completo de la arteria coronaria izquierda por una cúspide adherente de una válvula aórtica cuadricúspide ${ }^{10}$

\section{Diagnóstico}

El uso extensivo de la ecocardiografía, la imagen armónica, el equipo digital completo y el enfoque transesofágico permitieron verificar el diagnóstico antes y en un mayor número de casos, que las series quirúrgicas o automáticas. Por lo general es reconocible en muchos casos con ecocardiograma transtorácico. La vista de eje corto a nivel de la aorta permite una visualización precisa del número, engrosamiento y movilidad de las cúspides aórticas; El patrón clásico es una válvula aórtica comisural "en forma de $\mathrm{X}^{\text {" en la diástole en }}$ comparación con la "Y" en los trivalva. El Doppler color evalúa la presencia y la gravedad de la insuficiencia aórtica.

En algunos casos, el ecocardiograma transtorácico falla el diagnóstico, pero, al menos, aumenta la sospecha de cúspides anómalas. En estos casos, el ecocardiograma transesofágico es una herramienta precisa para definir el diagnóstico, demostrar las cuatro cúspides, su tamaño, la variante de clasificación y visualizar el posible desplazamiento del ostium coronario. La resonancia magnética y la tomografía computarizada tienen un valor diagnóstico muy alto, pero no se recomienda su uso en la práctica clínica $^{11}$.

\section{Historia Natural}

El número relativamente pequeño de válvulas cuadricúspides reportado en la literatura no permite conclusiones definitivas sobre la historia natural.
Esta anomalía se ha asociado con frecuencia con insuficiencia aórtica (75\% de los casos), se notificó una función normal de la válvula aórtica en el $16 \%$ de los pacientes, mientras que la estenosis valvular es poco frecuente. La progresión a regurgitación moderada-severa es rara antes de la edad adulta; la revisión más grande publicada incluyó 186 pacientes, con edades comprendidas entre 2 y 84 años; en esta serie, la incidencia más alta de función valvular normal fue en pacientes menores de 18 años y empeoramiento de la función después de 40 años. La mayor prevalencia de regurgitación aórtica severa (75\% de los casos) y cirugía (50\% de regurgitación aórtica) es en la quinta o sexta década.

A diferencia de la válvula aórtica bicúspide, la válvula cuadricúspide no está asociada a la dilatación de la aorta ascendente. De hecho, en casi todos los casos informados, la raíz aórtica y la aorta ascendente se encontraban dentro de los límites normales. Estos datos tienen importancia clínica y de gestión.

No existe una preferencia sexual significativa con respecto a la distribución de QAV, sino solo un ligero predominio masculino ${ }^{3}$.

Se encontró endocarditis infecciosa en el 1,4\% de los casos. Por lo tanto, se recomienda la profilaxis $^{12}$.

En un número no despreciable de casos (alrededor del $10 \%$ ), se asocia con ostium coronario colocado de forma anormal. Esta anomalía no clínicamente dañina tiene importancia práctica para la intervención quirúrgica para guiar al cirujano al enfoque más apropiado. En la literatura se publicaron casos de muertes como resultado de la obstrucción del ostium coronario por un anillo protésico aórtico ${ }^{13}$.

\section{Conclusión}

La válvula cuadricúspide es una anomalía rara. Su incidencia varía de $0.008 \%$ a $0.043 \%$ según la modalidad de diagnóstico. Con frecuencia, es un hallazgo aislado, pero puede coexistir más frecuente con ostium coronario de implantación anómala.

La ecocardiografía es una técnica muy precisa para el diagnóstico. El ecocardiograma transtorácico permite en muchos casos el diagnóstico y establece la gravedad de la insuficiencia aórtica, el tamaño y la función del ventrículo izquierdo, la presencia de lesiones cardíacas asociadas y el tamaño de la aorta ascendente. ecocardiograma transesofágico, sin embargo, es una herramienta más sensible que define todos los detalles poco claros en ecocardiograma transtorácico y visualiza correctamente los ostiums coronarios. 
La gran mayoría de los pacientes tienen una función anormal y a menudo requieren cirugía por disfunción de la válvula aórtica, más comúnmente por insuficiencia aórtica, generalmente en las décadas quinta y sexta. Como tal, los pacientes con válvula aórtica cuadricúspide requerirían una evaluación clínica cuidadosa y un seguimiento minucioso para su evaluar su evolución y planear la mejor terapéutica para el mismo.

\section{Bibliografía}

1. Simonds JP. Malformación congénita de aórtica y pulmonar válvulas A.m. J. Med. Sci.1923;166: 584-95.

2. Olson LJ, Subramanian R, Edwards WD. Patología Quirúrgica de insuficiencia aórtica pura: un estudio de 225 casos.Mayo Clin. Proc.1984;59: 835-41.

3. O. Tutarel The quadricuspid aortic valve J. Heart Valve Dis., 14 (2004), pp. 534-537.

4. Khan SK, Tamin SS, Araoz PA. Válvula aórtica cuadricúspide por Imagen de resonancia magnética cardíaca: reporte de un caso y revisión de la literatura.J. Comput. Ayudar. Tomogr2011;35: 637-41.

5. Daprati A, Generali T, Arlati F, et al. Válvula aórtica cuadricúspide plastia: ¿vale la pena repararlo como alternativa a la sustitución?Ana.Thorac Surg.2013;95: e7-8.

6. M.H. Brouwer, J.J. de Graaf, T. Ebel Congenital quadricuspid aortic valv Int. J. Cardiol., 38 (2) (1993), pp. 196-198.

7. L.E. Hurwitz, W.C. Roberts Quadricuspid semilunar valve Am. J. Cardiol., 31 (1973), pp. 623-626.

8. Jagannath $\mathrm{AD}$, Johri AM, et al. La válvula aórtica cuadricúspide: un informe de 12 casos y una revisión de la literatura. Ecocardiografía 2011 oct; 28 (9): 1035-40.

9. F. Robicsek, P.W. Sanger, H.K. Daugherty, et al. Congenital quadricuspid aortic valve with displacement of the left coronary orifice Am. J. Cardiol., 23 (1969), pp. 288.

10. H. Kurosawa, S.S. Wagenaar, A.E. Becker Sudden death in a youth. A case of quadricuspid aortic valve with isolation of origin of left coronary artery Br. Heart J., 46 (1981), pp. 211.

11. Hayakawa M, Asai T, Kinoshita T, Suzuki T Ann Thorac Cardiovasc Surg. 2014; 20 Supl (): 941-4.
12. Takeda N, Ohtaki et al. Endocarditis infecciosa asociada a válvula aórtica cuadricúspide. Jpn Heart J. 2003 mayo; 44 (3): 441-5.

13. Robicsek F, Sanger PW, Daugherty HK, Montgomery CC Válvula aórtica cuadricúspide congénita con desplazamiento del orificio coronario izquierdo.Coll Works Cardiopulm Dis. 1968 dic; 14 (): 87-90.

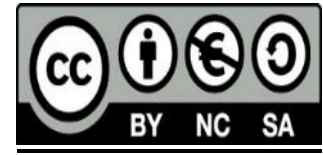

